EPJ Web of Conferences 116, 09002 (2016)

DOI: $10.1051 /$ epjconf/201611609002

(C) Owned by the authors, published by EDP Sciences, 2016

\title{
Design and mass production of the optical modules for KM3NeT-Italia project
}

\author{
Emanuele Leonora $^{a}$, Sebastiano Aiello, and Valentina Giordano on behalf of KM3NeT-Italia project \\ Istituto Nazionale di Fisica Nucleare, sezione di Catania, via S. Sofia 64, 95125 Catania, Italy
}

\begin{abstract}
The KM3NeT European project aims at constructing a $\mathrm{km}^{3}$ underwater neutrino telescope in the depths of the Mediterranean Sea. The first phase that is under construction will comprise eight tower-like detection structures (KM3NeT-Italia), which will form the internal core of $\mathrm{a} \mathrm{km}^{3}$-scale detector. The detection element of KM3NeT-Italia, the optical module, is made of a 13-inch pressure-resistant glass-vessel that contains a single 10-inch photomultiplier and the relative electronics. The design of the whole optical module, the main results obtained from the massive photomultipliers measurements, and the foremost phases of the mass production procedure performed at the production site of Catania are also presented.
\end{abstract}

\section{Introduction}

The KM3NeT European project aims at constructing a $\mathrm{km}^{3}$ underwater neutrino telescope in the depths of the Mediterranean Sea. Thanks to a dedicated funding by the Italian Ministry of Education, University and Research (MIUR) the construction of a first phase of the Italian KM3NeT node is under way. This detector will comprise eight tower-like detection units and 24 strings. The tower is composed by 14 storeys, each $8 \mathrm{~m}$ long, spaced out vertically of $20 \mathrm{~m}$. The detection element of the telescope, called optical module $(\mathrm{OM})$, is a pressure-resistant transparent glass vessel that contains a $10^{\prime \prime}$ photomultiplier and the electronics devices for power supply, signal read-out and transmission.

Each storey of the tower hosts 6 optical modules, arranged into three couples downgoing and horizontally at each extremity and at the storey-centre looking $45^{\circ}$ downwards. The optical module design, as well as each single component, was defined after an intense R\&D work on photomultipliers, high voltage supply circuit, optical gel and mechanical supports. During the optical module production phase, 750 10-inch R7081 Hamamatsu photomultipliers have been characterized by means of dedicated test-benches. Following a well-established production procedure, 700 optical modules were assembled at the INFN production site located in Catania, and their electrical and mechanical functionalities were tested by using properly test-benches and also a hyperbaric chamber. The paper describes the design of the optical module and its main components in Sect. 2, the main results obtained from the massive

\footnotetext{
a e-mail: emanuele.leonora@ct.infn.it
}

This is an Open Access article distributed under the terms of the Creative Commons Attribution License 4.0, which permits unrestricted use, distribution, and reproduction in any medium, provided the original work is properly cited. 


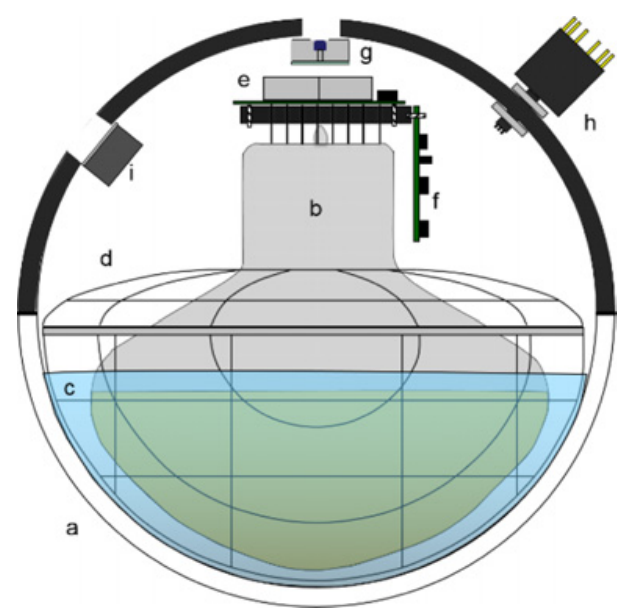

Figure 1. Schematic view of the main components of an optical module: (a) 13" glass sphere, (b) PMT, (c) optical glue, (d) mu-metal cage, (e) HV base, (f) FEM (Front End Module), (g) LED beacon, (h) 8-pin connector, (i) Manometer.

photomultiplier measurements in Sect. 3, and also the main phases of the mass production procedure in the last Sect. 4.

\section{The optical module design}

The optical module is composed by a 13 -inch pressure-resistant spherical glass-vessel produced by Benthos [1], which works as water and pressure protection for internal devices, ensuring also a good light transmission. Two hemi-spherical halves compose each vessel: a transparent one that supports the photo-detector, and a painted black one, which hosts the electronics. A single 10-inch photocathode photomultiplier, type R7081 produced by Hamamatsu [2], was chosen as photodetector, and it is optically and mechanically coupled to the transparent hemisphere by means of transparent glue Wacker SilGel 612 [3]. Inside the OM, a mu-metal cage, produced by ITEP [4], surrounds the PMT to shield it against the Earth's magnetic field. An active base produced by ISEG [5] supplies the PMT high voltage (HV). A custom designed [6] front-end electronic module (FEM), drives the HV base, digitizes the PMT signals, encodes and transmits the data to the external floor control module by means of an 8-pin SEA CON connector [7]. Inside the optical module there is a LED beacon system designed to emit calibrated light pulses for underwater calibrations and also a pressure meter to allow the check of the vacuum inside the sphere. Figure 1 shows schematically the OM components.

\section{The photomultiplier characterization phase}

The R7081 Hamamatsu photomultiplier has 10 stages and a 10-inch bialkali photocathode with a typical detection surface of about $500 \mathrm{~cm}^{2}$ and a quantum efficiency of about $25 \%$ at the spectral peak of $400 \mathrm{~nm}$. In the frame of the massive optical modules production, a batch of 750 photomultipliers was tested by means of a dedicated facility. The tests were performed on PMTs with the HV base already soldered, surrounded by the mu-metal cage, positioned into properly dark box where they were left for at least 8 hours before the measurements. The facilities developed to test the 750 PMTs consisted of 4 light-tight dark boxes, which allow automatized test on 8 PMTs per day. A software developed in LabView for acquisition and data analysis manages the DAQ system composed by two Digitizer 
Table 1. Measurements from a batch of 750 R7081 Hamamatsu PMTs.

\begin{tabular}{|l|c|c|c|}
\hline Parameters & $\begin{array}{c}\text { Required values } \\
\text { values }\end{array}$ & $\begin{array}{c}\text { Mean values } \\
\text { measured }\end{array}$ & $\begin{array}{c}\text { Value Ranges } \\
\text { measured }\end{array}$ \\
\hline Voltage at Gain 5E7 [V] & $<2000$ & 1648 & $1460 \div 1900$ \\
\hline Dark Count rate [Hz] & $<8000$ & 1128 & $274 \div 13200$ \\
\hline P/V ratio & $>2$ & 3.2 & $1.76 \div 4.5$ \\
\hline TTS FWHM [ns] & $<3.5$ & 2.4 & $1.98 \div 3.85$ \\
\hline Pre-Pulse [\%] & $<1$ & 0.18 & $0.04 \div 2$ \\
\hline Delayed Pulse [\%] & $<8$ & 5.65 & $4 \div 10.8$ \\
\hline Type 1 after pulse [\%] & $<3$ & 0.47 & $0.05 \div 4.71$ \\
\hline Type 2 after pulse [\%] & $<10$ & 7.3 & $3.1 \div 16.5$ \\
\hline
\end{tabular}

PCI-5153 produced by National Instruments [8], with a $500 \mathrm{MHz}$ bandwidth, 2 GSample/seconds sampling and 8-bit resolution.

Table 1 summarizes all the measurements from the whole batch of PMTs, together with the project specifications. As it can be seen, the results confirm that such PMTs comply with the project's requirements. Among the whole batch of 750 PMTs, only 14 PMTs were rejected, because one or more parameters didn't comply with the range required, mainly the fraction of type 2 after pulses, the peak to valley ratio and the dark count rate.

\subsection{Aging effects}

A study of the aging effects of the PMTs to measure the variations of the parameters with the operating time was performed for almost 3 years (equivalent operating time of 45 years in water, with an optical background of 1 photo-electron (pe) at $200 \mathrm{kHz}$ ). The measurements consisted of cycles of two different phases: an accelerated aging phase, illuminating the PMT under test with a 3-pe light pulses at $1 \mathrm{MHz}$, interposed with a characterization phase, which consisted of measurements of gain, time and charge characteristics and spurious pulses. Results showed that all the PMT's parameters did not change substantially during long operating periods apart the gain, which had a maximal variation of about $30 \%$ [9].

\subsection{The Earth's magnetic field effects on PMTs}

Performances of large area photomultipliers are influenced by magnetic fields, because of the long trajectories of electrons from the photocathode to the anode. Variations on PMT parameters were measured with and without the mu-metal cage changing the PMT orientations with respect to the Earth's magnetic field. All the results confirmed that the performances of large area PMTs without any magnetic shield is affected by changes of its orientation with respect to the Earth's magnetic field, with variations of about $30 \%$ for the gain and over $40 \%$ for the detection efficiency and also for the peak to valley ratio. The use of a mu-metal cage as magnetic shielding considerably reduces these variations [9]. A magnetic shield is therefore mandatory in optical modules with large are photomultipliers.

\section{The main phases of the production procedure}

Following a well-established production procedure, almost 700 optical modules were assembled at the INFN integration site located in Catania. For the outgassing phase of the optical gel and for the closure of the two hemispheres of the optical modules, four vacuum boxes were constructed $\left(1 \times 1 \times 1 \mathrm{~m}^{3}\right)$, in which the absolute vacuum decreases to over $300 \mathrm{mbar}$ in less than $2 \mathrm{~min}$. To reduce the effects of 
humidity, each box was equipped with a system of Nitrogen flux. Schematically, the main phases of the assembly procedure can be described as follows:

- soldering of the high voltage supply base on the leads of the PMTs;

- testing of each PMT with the base already soldered, as described in Sect. 3. Only the PMTs that fully complied with the requirements were assembled into the optical modules;

- the HV base and the leads of each PMT were covered with a liquid coating (that dries in about 24 hours) to isolate all the electrical contacts;

- the transparent hemisphere where the PMT will be glued was cleaned carefully with optical paper and ethanol, and positioned into the vacuum box;

- the hemispherical part of the mu-metal cage was cleaned carefully with optical wipes and positioned into the glass hemisphere;

- one out-gassing cycle into the vacuum box with an under pressure of $400 \mathrm{mbar}$, followed by an air re-entry phase, made the elements ready for the optical gel pouring

- mixing of the two components of the optical gel, A and B, with a ratio of 100:60, with a controlled mixer with 120 turn/minutes, for 5 minutes, to prepare the required 1.5 litre of optical glue;

- pouring of the optical glue into the hemisphere, with the mu-metal cage already in position;

- two outgassing phases to accelerate the extraction of the air bubbles inside the gel;

- by means of proper tools, the PMT was positioned and immersed into the gel inside the hemisphere;

- three degassing phases, each one followed by a phase of Nitrogen re-entry to remove all the residual air bubbles and reduce humidity inside the glue;

- polimerization of the optical gel, performed at room temperature and atmospheric ambient for at least 12 hours;

- the Front End Electronic board was mounted by means of proper PVC structures, and connected to the PMT anode by a coaxial cable and to the HV base by a flexible ribbon cable;

- glueing of the LED beacon system by means of black silicone in a transparent window opened in the black hemisphere and its connection to the FEM board by a twisted cable;

- two sacks of Silica gel of $5 \mathrm{~g}$ were positioned into the OM, to reduce the humidity inside;

- to close the OM, the two hemispheres were positioned into the vacuum box. A wedge inserted trough the two hemispheres maintains open the OM for a 10-minute phase of Nitrogen flux, to reduce the internal humidity to less than $2 \%$. When the hygrometer inside the box shows the expected value, an electric system manually driven removes the wedge, and the two hemispheres joined together;

- an under-pressure of 400 mbar and a following Nitrogen re-entry phase close the whole sphere;

- one turn of sealant Terostat-81 $20 \times 2 \mathrm{~mm}$ and three following turns of 50-mm tape Scotchrap $3 \mathrm{M}$ all-weather corrosion protection applied at the junction of the two hemispheres sealed definitely the optical module. The under pressure inside is constantly checked by the manometer;

- a proper test-bench tested the functionality for each OM. A hyperbaric chamber also tested the watertight for random OMs, up to 350 bars.

\section{Conclusions}

A 13-inch diameter optical module with a large 10-inch PMT was designed as the detection element of the KM3NeT-Italia project. A batch of 750 R7081 Hamamatsu photomultipliers was characterized. Following a well-established production procedure, almost 700 optical modules were assembled at the INFN integration workshop located in Catania. The functionality was tested both from electrical and mechanical point of view by using proper test-benches and a hyperbaric chamber. They will be soon mounted in the tower-like detection units and deployed in the deep-sea site. 


\section{References}

[1] Teledyne Benthos web site, http://www. benthos.com

[2] Hamamatsu web site, http://www.hamamatsu.com

[3] Wacker Chemie AG, http://www .wacker.com/cms/en/home/index.jsp

[4] Institute for Theoretical and Experimental Physics, Moscow, http://www. itep.ru

[5] SEG web site, http://www.iseg-hv.com

[6] C. Nicolau, proceeding of this conference

[7] SEA CON web-site: http://seaconword.com

[8] National Instrument web site: http://www.ni.com

[9] E. Leonora et al., 2015 JINST 10 T11003 